\title{
THE EFFECTS OF LEAD-VEHICLE SIZE ON DRIVER FOLLOWING BEHAVIOR: IS IGNORANCE TRULY BLISS?
}

\author{
James R. Sayer, Mary Lynn Mefford \\ Human Factors Division \\ University of Michigan Transportation Research Institute \\ Ann Arbor, MI 48109-2150 \\ E-mail: jimsayer@umich.edu \\ Ritchie W. Huang \\ Information Technology Research Division \\ Los Angeles Office of Honda R\&D Americas, Inc.
}

\begin{abstract}
Summary: The objective of this study was to examine whether size of a lead vehicle (passenger car or light truck) affects the distance at which following vehicles travel. Naturalistic following data were collected from drivers using instrumented passenger cars in place of their own vehicles. The results show that these drivers followed light trucks at shorter distances than they followed other passenger cars by an average of $5.6 \mathrm{~m}$, or .19 $\mathrm{s}$ in headway time margin, but at the same velocities and range-rates. This result is discussed in the context of a passenger car driver's ability to see beyond a lead vehicle to assess, and respond to, the status of traffic downstream. The results of this study suggest that knowing the state of traffic beyond the lead vehicle, even by only one additional vehicle, affects gap length. Specifically, it appears that when dimensions of lead vehicles permit other drivers to see through, over, or around them, drivers maintain significantly longer (i.e., safer) distances.
\end{abstract}

\section{INTRODUCTION}

This study was designed to contribute to an understanding of how light trucks might influence gap maintenance behavior when they precede passenger cars. Vehicles that are classified as light trucks include sport utility vehicles, minivans, passenger vans, and pickup trucks. It was expected, a priori, that passenger car drivers would leave longer gaps between the front of their vehicle and the rear of a lead vehicle when the lead vehicle was a light truck, as the size of a light truck would more likely obstruct the view of passenger car drivers (Figure 1). It is not unusual for drivers to report, at least anecdotally, that they avoid following large vehicles. The basis for aversion to following large vehicles is generally attributed to a driver's recognition of how visual information regarding the forward scene may be obstructed by a large vehicle. The rationale for leaving longer gaps behind light trucks would be to allow more time to respond to events for which drivers have little or no advance notice — other than the actions of the vehicle directly in front.

In order to test this hypothesis, data from the 1996-97 Intelligent Cruise Control Field Operational Test (ICC FOT) were examined (a detailed description of this study can be found in Fancher, Ervin, Sayer, Hagan, Bogard, Bareket, Mefford, and Haugen, 1998). In the ICC FOT, 108 participants drove an instrumented passenger car as their personal means of transportation. During this period, the vehicles were put into naturalistic use, with almost no constraint on 
where, when, or how participants drove. Each driver was free to choose between operating manually or with conventional cruise control during the first week, and between manual operation and ICC during the second (or subsequent) weeks. The bulk of the accumulated mileage, $176,000 \mathrm{~km}$, was under manual control. Digital video of the forward scene was continuously collected, with samples being saved at regular intervals. By examining this video and associated data it could be determined what effects, if any, lead-vehicle size (passenger car or light truck) had on a passenger car driver's gap maintenance. For the purpose of this paper, driver characteristic variables such as age and gender are not reported. A more thorough analysis of this data can be found in Sayer, Mefford \& Huang (2000).

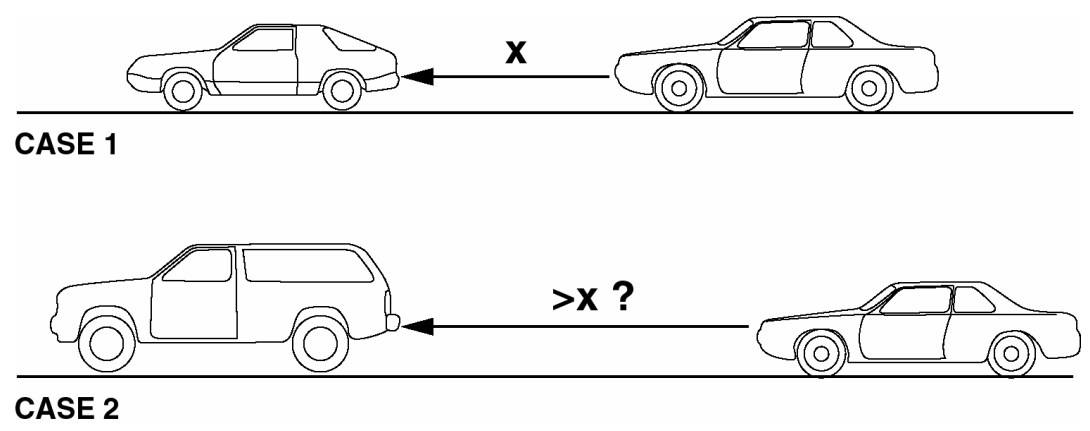

Figure 1. Hypothesized following relationship between light trucks and passenger cars

\section{METHOD}

Each of ten vehicles involved in this FOT was equipped with an ICC system and supporting equipment — such as a GPS and a data acquisition system. All vehicles used infrared sensors mounted in the front grill as a means of distance measurement. Data were continuously collected whenever the car was in operation. In each research vehicle, a CCD video camera was mounted on the inside of the windshield, behind the rearview mirror. The camera continuously digitized and stored captured video to internal buffers in the data acquisition system. The system saved 2.5-second exposures to a file every 5 minutes or 10 minutes, depending on the length of a driver's participation in the study. Each image frame contained two interlaced fields (243 rows by 512 pixels). The video software sampled a stripe from the even field, which was 64 rows by 512 pixels, every six frames, or 0.1 seconds. The video files were later converted to QuickTime movies. The images were doubled in height to recapture the original aspect ratio and compressed. An example of an individual frame from a video is provided in Figure 2.

The numerical data for range to the lead vehicle and velocity were collected at a rate of $10 \mathrm{~Hz}$ from the infrared distance sensor and the vehicle's engine control unit. Range rate and headway time were computed on-line and stored at the same sampling rate. A database containing 20,768 video exposure files and associated numerical data was queried. Active following was initially defined as: the host vehicle was being operated manually, an object was detected by the infrared sensor, the velocity of the host vehicle was greater than, or equal to, $64 \mathrm{~km} / \mathrm{h}(40 \mathrm{mph})$, and range-rate, the rate of change of range, was within $+/-1.5 \mathrm{~m} / \mathrm{sec}(5 \mathrm{ft} / \mathrm{sec})$. The minimum velocity criterion was selected in an attempt to eliminate scenarios where frequent stopping might be observed. Range rate was limited to $+/-1.5 \mathrm{~m} / \mathrm{sec}(5 \mathrm{ft} / \mathrm{sec})$ in order to eliminate scenarios in which the host vehicle was either rapidly closing in on, or falling back from, a lead vehicle. Relatively large values of range rate indicated that the velocities of the host and lead 
vehicle were likely too different to be consistent with "active following," and may instead have been the beginning or the end of a passing maneuver.

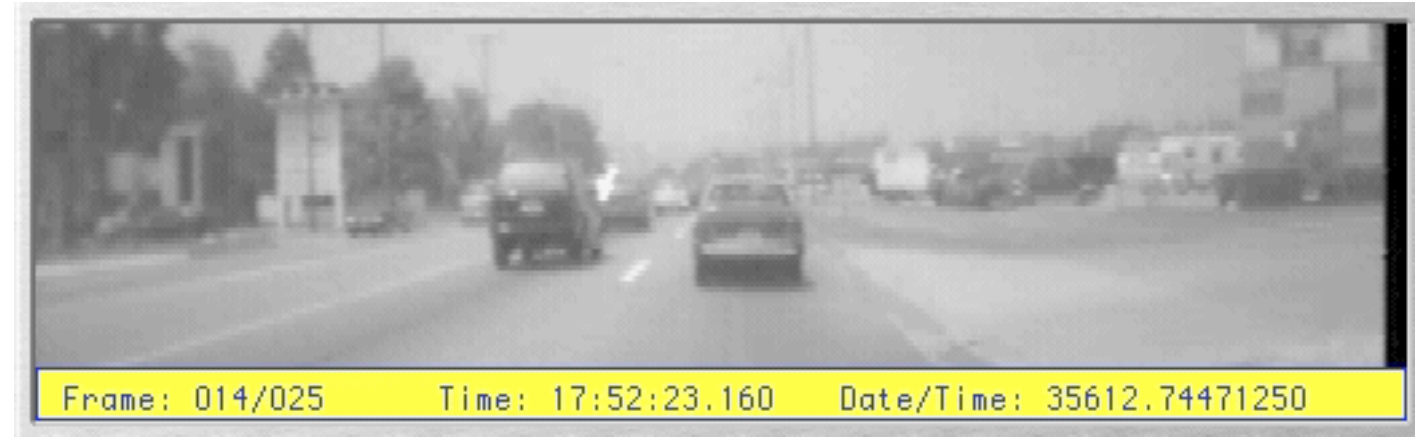

Figure 2. Example frame from an exposure video showing a passenger car as the lead vehicle

A total of 2,843 video clips satisfied the above criteria. Each clip was then viewed in order to apply several additional, visually based, limiting criteria. The criteria imposed based on visual inspection were weather conditions, time of day, and lateral movement (specifically lane changes or merges). Only clips that were recorded during daylight with no precipitation and no lateral motion by either the lead or host vehicles were selected for further analyses. These criteria were imposed because both low illumination and poor visibility seriously inhibited the accurate classification of lead vehicles, and instances of merging, passing, or cut-ins were dramatically different from following events. As a result, only 1,845 of the video clips remained. Accurate classification was possible at a maximum of approximately 3 seconds of headway time at highway speeds. Passenger cars were defined as those vehicles that were short enough in height to permit the following driver, in a passenger car, to see through or around them. Light trucks (sport utility vehicles, minivans, vans, pickup trucks) were defined as larger/taller, vehicles that did not permit the following driver, in a passenger car, to see through them). Only three motorcycles were observed and treated as passenger cars in the analyses. Commercial vehicles were not included in these analyses.

\section{RESULTS}

Because data were collected from naturalistic driving there were unequal numbers of observations across conditions. Data from the 1,845 video clips were reduced by identifying participants with a minimum of two observations for each of the two vehicle sizes and the mean for each size was calculated separately for each of the four dependent measures (range, velocity, headway time margin and range-rate). As a result 1,698 observations remained, representing data from 70 drivers. Because of the unequal number of observations making up each participant's mean, a weighting of the means was performed and a final adjustment was made to the mean weighted values prior to the analyses (see Sayer, et al., 2000).

Four analyses of variance were performed using the mean weighted values of range, velocity, headway time margin, and range-rate as the dependent measures. In each of the four ANOVAs the within-subjects variable was lead-vehicle size (passenger car or light truck). The between- 
subjects factors were participant age (three levels), and participant gender (two levels). Due to space constraints, findings for the between-subjects variables are not reported here.

The main effects of vehicle size (passenger car or light truck) on the dependent measures of range and headway time margin were statistically significant, $F(1,64)=22.8, p<.001$ and $F(1,64)=17.9, p<.001$, respectively. Table 1 shows that participants followed light trucks more closely than passenger cars by an average of $5.6 \mathrm{~m}$. Yet neither velocity nor range-rate systematically varied with lead-vehicle size. Values of headway time margin were similarly decreased when following light trucks $(1.41 \mathrm{~s})$ relative to passenger cars $(1.60 \mathrm{~s})$. This effect was consistently observed across all levels of between-subjects factors. The lack of a significant effect of vehicle size on either velocity or range-rate suggests that exposure conditions were comparable for both levels of vehicle size.

Table 1. Means and Standard Errors of Range and Headway Time Margin (HTM)

\begin{tabular}{cccccc} 
& \multicolumn{2}{c}{ Range $(\mathrm{m})$} & & \multicolumn{2}{c}{ HTM $(\mathrm{s})$} \\
\cline { 2 - 3 } \cline { 5 - 6 } Vehicle Size & Mean & Std. Error & & Mean & Std. Error \\
\hline \hline Light Truck & 34.0 & 0.88 & & 1.41 & 0.04 \\
\hline Passenger Car & 39.6 & 1.34 & & 1.60 & 0.05 \\
\hline
\end{tabular}

\section{DISCUSSION}

Maintaining shorter gaps behind larger vehicles may seem counterintuitive as well as contrary to what drivers report anecdotally (Green and Yoo, 1999). Nonetheless, because there were no differences in velocity or range-rate associated with vehicle size in these analyses, it appears that exposure conditions were comparable when following both passenger cars and light trucks. Furthermore, the proportion of passenger cars and light trucks observed, 65\% and 35\% respectively, is thought to be reasonably representative of the distribution of vehicles on the road. What then could explain the difference in gap length/headway time margin associated with leadvehicle size observed in these data? Potential explanations are discussed.

Large vehicles take longer to stop or their velocities fluctuate less. One possible explanation of the observed difference in gap length/headway time margin is that drivers believe that light trucks have lower rates of deceleration (i.e., their braking performance is less than a passenger car). If light trucks were believed to have lower rates of deceleration, then following drivers would feel they had more time and distance in which to bring their own vehicles to a stop subsequent to lead-vehicle braking. Similarly, if the velocity of light trucks was perceived by drivers to fluctuate less than passenger cars, then drivers might believe that maintaining shorter gaps behind light trucks was not inherently unsafe in comparison to following passenger cars, for which velocities were perceived to fluctuate more widely at the same distance.

Ignorance is bliss. In an annotated bibliography on driver passing/overtaking behavior, Berggrund (1973) reported the results of two Swedish studies conducted by Åhman (1968 and 1972). The results of these two on-road studies lend support to the explanation that drivers do not necessarily worry about what they cannot see with regard to road conditions ahead. Åmman's research suggests that uncertainty of the road condition does not appear to inhibit attempts at passing/overtaking behavior. In a similar fashion, not being able to see traffic beyond the lead 
vehicle due to the size of the lead vehicle reduces a driver's awareness of traffic conditions ahead, but may not inhibit close following behavior. In some respects, limiting a driver's monitoring of forward traffic to a single lead vehicle lends itself to applying a much simpler strategy for the following driver. Rather than having to monitor multiple vehicles in the same lane, possibly through successive layers of automotive glass, a driver might reduce his/her effort by concentrating solely on, and responding only to, the immediate lead vehicle - as it may very well be the only vehicle in their lane for which information is available.

\section{CONCLUSIONS}

The results of the present study suggest that lead vehicles that are larger than passenger cars are followed more closely than passenger cars, at least by drivers of passenger cars. Given that it is unlikely we will see a reduction in the sale of light trucks relative to passenger cars in the near future, it is important to continue to investigate gap maintenance behavior associated with leadvehicle size. Although it is necessary that following drivers be able to see the stop lamps on lead vehicles, in and of itself that may not be sufficient for safe driving. The results of this study suggest that knowing the state of traffic beyond the lead vehicle affects gap maintenance. Specifically, it is suggested that when a lead vehicle permits a following driver to see through, over, or around it, drivers maintain significantly longer (i.e., safer) following distances. However, the rationale to account for such behavior remains unknown.

\section{ACKNOWLEDGEMENTS}

The authors would like to acknowledge the UMTRI Industry Affiliation Program for Human Factors in Transportation Safety and the Undergraduate Research Opportunity Program, both at the University of Michigan, for their support of this work.

\section{REFERENCES}

Berggrund, U. (1973). Annotated bibliography of overtaking behavior. Courbevoie, France: International Drivers' Behaviour Research Association.

Fancher, P. S., Ervin, R. D., Sayer, J. R., Hagan, M., Bogard, S., Bareket, Z., Mefford, M. L., and Haugen, J. (1998). Intelligent cruise control field operational test. (Report No. DOT HS 808 849). Ann Arbor, MI: The University of Michigan Transportation Research Institute.

Green, P. and Yoo, H. (1999). Driver behavior while following cars, trucks, and buses (Report No. UMTRI-99-14). Ann Arbor, MI: The University of Michigan Transportation Research Institute.

Sayer, J. R., Mefford, M. L., and Huang, R. W. (2000). The Effect of Lead-Vehicle Size on Driver Following Behavior (Report No. UMTRI-2000-15). Ann Arbor, MI: The University of Michigan Transportation Research Institute. 